\section{Differential diagnosis of intracoronary lesions}

A 39-year-old man presented with a non-ST segment elevation myocardial infarction which was treated with thrombolysis, and he made an uneventful recovery. His only risk factor was smoking for 5 years. Echocardiogram showed an ejection fraction of $55 \%$. He denied previous episodes of chest pain, unusual childhood illnesses and inflammatory diseases. A month later he underwent coronary angiography (figures 1-3). All

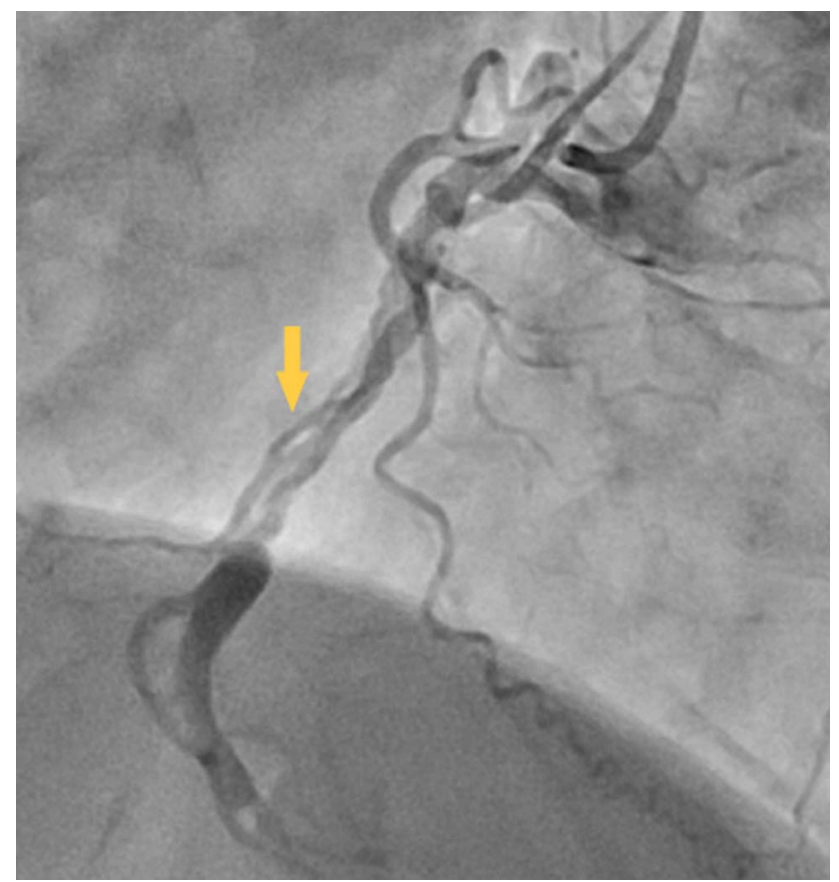

Figure 1 Right coronary artery injection; arrow indicates linear intracoronary lesion.

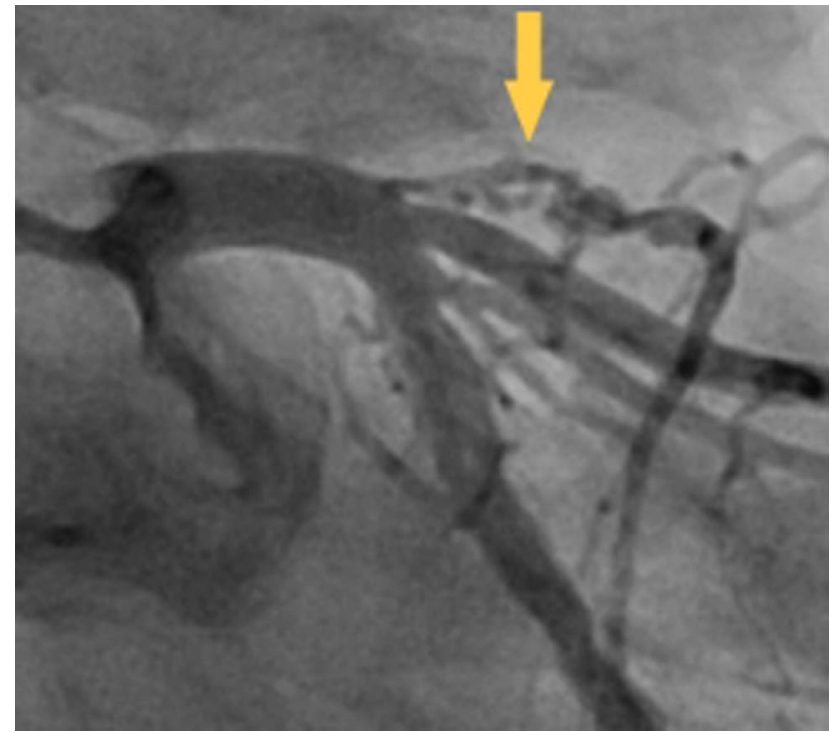

Figure 2 Left coronary artery injection; arrow indicates linear lesion in the proximal left anterior descending artery prior to occlusion.

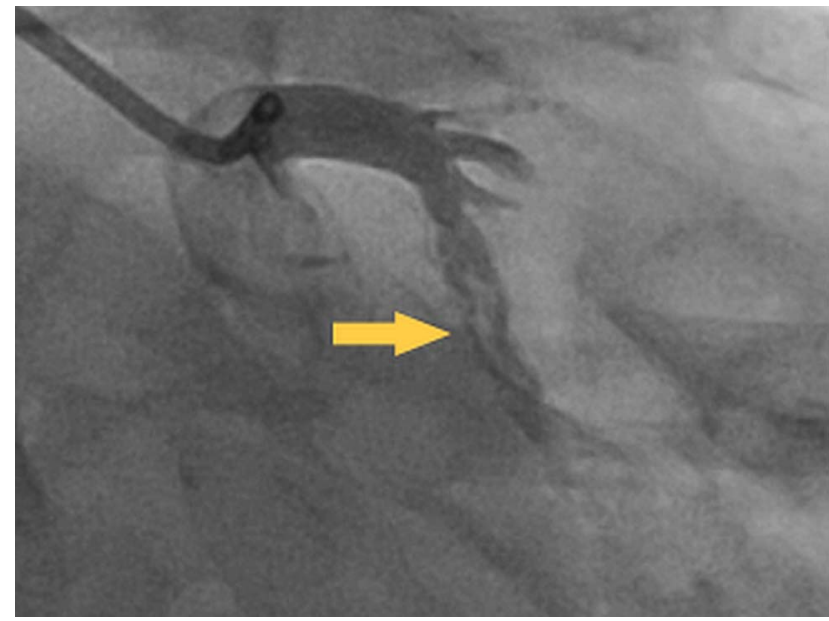

Figure 3 Left coronary artery injection; arrow indicates intracoronary lesion in the circumflex artery.

three coronary arteries had similar lesions. Auto antibody screening was negative, inflammatory markers were insignificant and CT scans of his chest and abdomen were normal. He underwent an uneventful coronary artery bypass surgery.

\section{DISCUSSION}

The linear nature of the lesion rules out intracoronary thrombus (also mobile) and atherosclerosis. Spontaneous coronary artery dissection (SCAD) is rare and presentation relates to the degree of ischaemia and will dictate any intervention required, ${ }^{1}$ whereas woven coronary artery anomaly (WCAA) is extremely rare and is characterised by the formation of multiple channels in any coronary artery with a Thrombolysis in Myocardial Infarction Study Group (TIMI-III) blood flow, with these channels joining distally; it is thought to be benign due to the normal distal blood flow. ${ }^{2}$

In this case, the right coronary artery and the left anterior descending artery became occluded beyond the lesions, whereas the circumflex had normal distal flow. This case is interesting as it may be all three arteries have SCAD or a combination of WCAA and SCAD, which has not previously been reported.

\section{Cornelia S Carr, Akhlaque Bhat, Abdulaziz M Alkhulaifi}

Department of Cardiothoracic Surgery, Heart Hospital, Doha, Qatar

Correspondence to Dr Cornelia Carr, Cardiothoracic Surgery, Heart Hospital, Doha 3050, Qatar; noahalkh@gmail.com

Contributors All authors contributed equally to the production of this manuscript.

Competing interests None.

Patient consent Obtained.

Ethics approval MRC Qatar approved showing of X-ray images.

Provenance and peer review Not commissioned; internally peer reviewed.

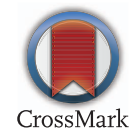

To cite Carr CS, Bhat A, Alkhulaifi AM. Heart Asia 2014;6:121. doi:10.1136/ heartasia-2014-010552

Heart Asia 2014;6:121. doi:10.1136/heartasia-2014-010552

\section{REFERENCES}

1 Tweet MS, Hayes SN, Pitta SR, et al. Clinical features, management, and prognosis of spontaneous coronary artery dissection. Circulation 2012;126:579-88. 